\title{
An adaptive local-global multiscale finite volume element method for two-phase flow simulations
}

\author{
L.J. Durlofsky, ${ }^{\text {a }}$ Y. Efendiev, ${ }^{b}$ V. Ginting ${ }^{\mathrm{c}, 1}$ \\ ${ }^{a}$ Department of Petroleum Engineering, Stanford University, Stanford, CA \\ 94305-2220 \\ ${ }^{\mathrm{b}}$ Department of Mathematics, Texas A\&M University, College Station, TX \\ 778 $433-3368$ \\ ${ }^{\mathrm{c}}$ Institute for Scientific Computation, Texas A $\mathscr{E}_{M}$ University, College Station, TX \\ 778 $433-3368$
}

\begin{abstract}
Multiscale solution methods are currently under active investigation for the simulation of subsurface flow in heterogeneous formations. These procedures capture the effects of fine scale permeability variations through the calculation of specialized coarse scale basis functions. Most of the multiscale techniques presented to date employ localization approximations in the calculation of these basis functions. For some highly correlated (e.g., channelized) formations, however, global effects are important and these may need to be incorporated into the multiscale basis functions. This can be accomplished using global fine scale simulations, but this may be computationally expensive. In this paper an adaptive local-global technique, originally developed within the context of upscaling, is applied for the computation of multiscale basis functions. The procedure enables the efficient incorporation of approximate global information, determined via coarse scale simulations, into the multiscale basis functions. The resulting procedure is formulated as a finite volume element method and is applied for a number of single and two-phase flow simulations of channelized two-dimensional systems. Both conforming and nonconforming procedures are considered. The level of accuracy of the resulting method is shown to be consistently higher than that of the standard finite volume element multiscale technique based on localized basis functions determined using linear pressure boundary conditions.
\end{abstract}

Key words:

PACS:

$\overline{1}$ Current Address: Department of Mathematics, Colorado State University, Fort

Preprint submitted to Elsevier Science

20 April 2006 


\section{Introduction}

The high degree of variability and multiscale nature of formation properties such as permeability pose significant challenges for subsurface flow modeling. Geological characterizations that capture these effects are typically developed at scales that are too fine for direct flow simulation, so techniques are required to enable the solution of flow problems in practice. Upscaling procedures have been commonly applied for this purpose and are effective in many cases (see $[19,17,11,12]$ for reviews and discussion). More recently, a number of multiscale finite element (e.g., $[14,7,3,4,1,2,9])$ and finite volume $[15,16]$ approaches have been developed and successfully applied for problems of this type.

As discussed in [12], upscaling methods and multiscale numerical techniques (as applied within the context of subsurface flow modeling) have many similarities and some important differences. Upscaling techniques provide coefficients, which are typically computed in a pre-processing step, for coarse scale equations of prescribed analytical forms. In multiscale methods, the coarse scale equations are formed numerically and fine scale information may be carried throughout the simulation and used at various stages. In multiscale procedures for subsurface flow applications, different grids are often used for flow and transport computations.

Most multiscale methods presented to date have applied local calculations for the determination of basis functions (or, in the case of variational multiscale methods [3,4], subgrid integrals). Though effective in many cases, global effects can be important for some problems. The importance of global information has been illustrated within the context of upscaling procedures in recent investigations $[13,6,5]$. These studies have shown that the use of global information in the calculation of the upscaled parameters can significantly improve the accuracy of the resulting coarse model. In [6,5], the global information was computed at the coarse scale (for reasons of computational efficiency), while in [13] fine scale global information was used.

Within the context of multiscale procedures, a few investigations have applied global information in the construction of basis functions. Aarnes et al. $[1,2]$ considered the use of both local and global information in their mixed finite element multiscale procedure and observed improved results when global effects were incorporated. Efendiev et al. [9] recently developed a multiscale finite volume element technique in which the basis functions are computed using the global pressure solution. They also demonstrated clear improvements in accuracy over a standard (local) procedure for a number of two-phase flow problems. Although these studies demonstrate the benefit of global information within a multiscale finite element framework, these approaches are po-

Collins, CO 80523-1874 
tentially limited by the need for global fine scale solutions. This might not be problematic if the boundary conditions do not change during the course of the global two-phase flow simulation, in which case only one global solution is required. However, if there are frequent changes in well rates or if new wells are introduced over the simulation time frame, as is common in practice, the global solutions could represent a large computational burden.

The goal of this paper is to develop a multiscale finite volume element method that uses global information at the coarse scale in the determination of the basis functions. As such, the method combines ideas from local-global upscaling techniques and previous multiscale finite volume element procedures. We will show that this approach provides improved accuracy relative to standard local procedures that use linear boundary conditions in the determination of the basis functions, though the accuracy is not in general as high as that achieved when global fine scale information is used. The method does, however, provide for a more efficient way of incorporating global information into the multiscale procedure. We describe both conforming and nonconforming multiscale finite volume element methods.

This paper proceeds as follows. We present the governing equations for twophase flow in Section 2. In Section 3 we describe adaptive local-global upscaling and then develop the adaptive local-global multiscale finite volume element procedure. Numerical results for a number of two-dimensional systems are presented in Section 4. We close with concluding remarks in Section 5.

\section{Governing equations}

We consider viscous-dominated two-phase flow in heterogeneous subsurface formations. The effects of gravity, capillary pressure and fluid and rock compressibility are neglected. The accurate coarse scale modeling of this simplified system is the basis for the modeling of more general systems, involving additional effects such as compressibility, so the approaches developed here can be expected to have wider applicability. The two phases are designated water (aqueous phase, subscript $w$ ) and oil (nonaqueous phase liquid, subscript $o$ ).

Darcy's law for each phase is given by:

$$
\mathbf{u}_{j}=-\frac{k_{r j}(S)}{\mu_{j}} \mathbf{k} \cdot \nabla p,
$$

where $\mathbf{u}_{j}$ is the Darcy phase velocity, $\mathbf{k}$ is the permeability tensor (taken as diagonal in this work), $k_{r j}$ is the relative permeability to phase $j(j=o, w), S$ is the water saturation (volume fraction), $p$ is pressure and $\mu_{j}$ is the viscosity 
of phase $j$. Defining the total velocity $\mathbf{u}$ as $\mathbf{u}=\mathbf{u}_{w}+\mathbf{u}_{o}=-\lambda(S) \mathbf{k} \cdot \nabla p$, where $\lambda$ is the total mobility given by:

$$
\lambda(S)=\frac{k_{r w}(S)}{\mu_{w}}+\frac{k_{r o}(S)}{\mu_{o}}
$$

and noting that $\nabla \cdot \mathbf{u}=-q$, gives the pressure equation:

$$
\nabla \cdot(\lambda(S) \mathbf{k} \cdot \nabla p)=q
$$

where $q=q_{w}+q_{o}$ is the total source term. The mass conservation equation for water can be expressed by noting that $\mathbf{u}_{w}=\mathbf{u} f(S)$, which gives the hyperbolic saturation equation:

$$
\phi \frac{\partial S}{\partial t}+\mathbf{u} \cdot \nabla f(S)=-q_{w}
$$

where we have used the fact that $\mathbf{u}$ is incompressible. The Buckley-Leverett flux function $f(S)$ is given by:

$$
f(S)=\frac{k_{r w}(S) / \mu_{w}}{k_{r w}(S) / \mu_{w}+k_{r o}(S) / \mu_{o}} .
$$

The above equations define the fine scale model. A single set of relative permeability curves is assumed to apply to the entire model. In subsequent descriptions, pressure and flow rate variables will be considered to be dimensionless.

\section{Numerical procedures}

In this section we first briefly describe the adaptive local-global upscaling procedure and then incorporate this concept into the multiscale finite volume element formulation. Both conforming and nonconforming procedures are considered. Some discussion as to why the adaptive local-global approach improves upon the standard method is then presented.

\subsection{Overview of adaptive local-global upscaling}

The basic idea of adaptive local-global (ALG) upscaling is to use global pressure information computed on the coarse scale for the prescription of boundary conditions for the upscaling calculations. The method improves upon local or extended local (i.e., oversampled) procedures because nonlocal effects enter into the calculation of coarse scale quantities. ALG procedures are not expected to be as accurate as global methods that use fine scale solutions (e.g., 
[13]), though ALG upscaling does avoid the need for any global fine scale solutions and will therefore provide enhanced efficiency, particularly in cases with changing global boundary conditions.

In the original finite volume implementation of local-global upscaling ([6]), both permeability and direct transmissibility upscaling were considered (upscaled permeability and transmissibility are designated $\mathbf{k}^{*}$ and $T^{*}$ respectively). Transmissibility, which is associated with block interfaces, is essentially the numerical analog of permeability and is the coefficient that relates the inter-block volumetric flow to the difference in block pressures (for a twopoint flux approximation). For highly heterogeneous systems, as considered in this paper, direct transmissibility upscaling was found to provide more accurate coarse models than permeability upscaling $[18,6]$. We now briefly describe ALG transmissibility upscaling, though the approach is similar for permeability upscaling.

The adaptive local-global upscaling procedure is illustrated in Fig. 1 (this figure is adapted from [5]). The first step entails the calculation of upscaled transmissibility (designated $T_{0}^{*}$ ) using a standard local method. Next, using these $T_{0}^{*}$, the global problem is solved subject to the actual global boundary conditions. The global pressure solution is then used to prescribe boundary conditions for the local determination of improved (or adapted) transmissibilities, designated $T_{1}^{*}$. These transmissibilities are computed using an extended local procedure with boundary conditions determined through interpolation of the global pressure solution (see Fig. 1b). This procedure is continued until some stop criteria are reached, such as small changes in pressure or flow rates from one iteration to the next. Typically only a few iterations are required to achieve a self-consistent solution. Wells are also treated within the general method, as indicated in Fig. 1c (see [5] for details). Because the upscaled transmissibilities are computed using global information, they are specifically "adapted" for a particular global problem. This distinguishes this "adaptive" local-global upscaling from a similar procedure ([6]) that uses generic (rather than specific) global pressure data.

A thresholding procedure is introduced into ALG upscaling for efficiency and to avoid the calculation of anomalous (e.g., negative) properties. This thresholding is incorporated as follows. At each iteration $\nu$, for a particular block to block connection, modified $T^{*}$ (designated $T_{\nu}^{*}$ ) are computed only if the flow rate exceeds a prescribed threshold value (criteria based on quantities other than flow rate could also be applied). This limits the number of $T_{\nu}^{*}$ to be recomputed to a fraction of the total and avoids the use of the global solution in regions where gradients are too small to provide reliable property estimates. In these regions, we simply use the previous estimate $T_{\nu-1}^{*}$.

If global boundary conditions change during the simulation, the $T^{*}$ can be 


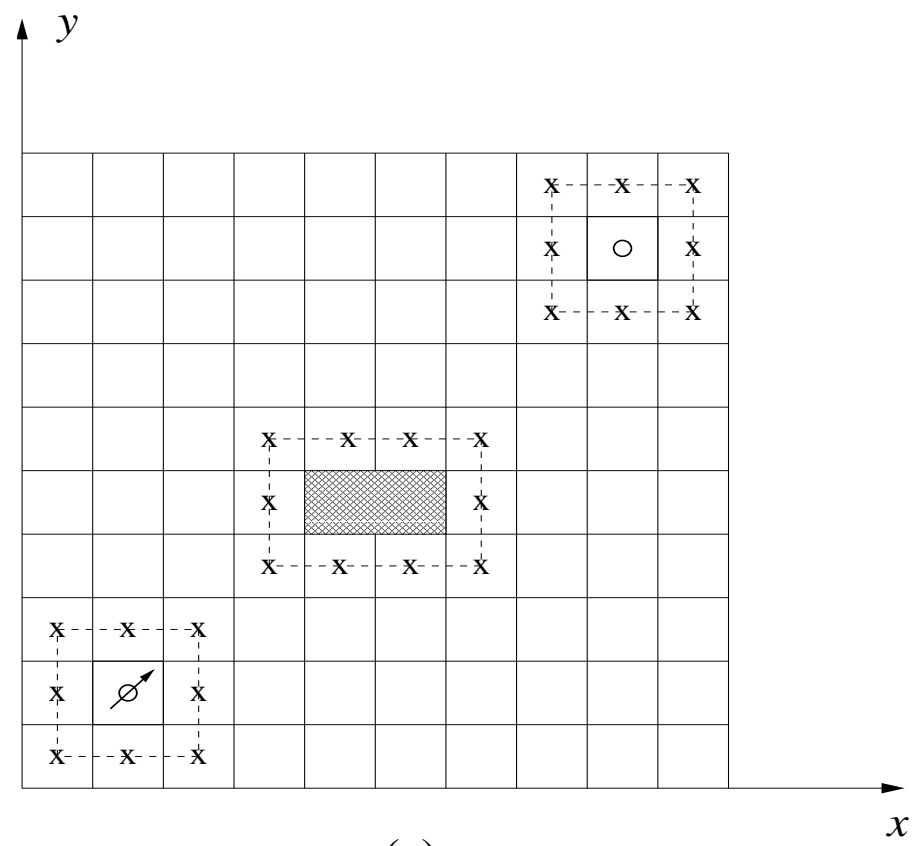

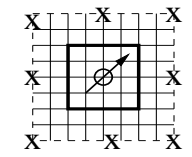

(c)

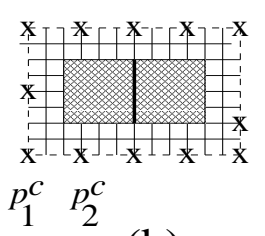

(b)

(a)

Fig. 1. Schematic illustration of the adaptive local-global transmissibility upscaling procedure.

recomputed. Again, because of the use of thresholding, the fraction of $T^{*}$ that must be recomputed is relatively small. This renders the overall procedure quite efficient and enables the incorporation of time-dependent global information into the upscaled model.

\subsection{Formulation of adaptive local-global multiscale finite volume element method (ALG-MsFVEM)}

Our objective here is to introduce the adaptive local-global upscaling procedure described above into the multiscale finite volume element framework. The main idea of multiscale finite element methods, first introduced in [14], is to incorporate fine scale information into the finite element basis functions through use of the solutions of local problems. In this paper, we apply ideas from localglobal upscaling for the construction of these basis functions. In this way, the basis functions will contain global information. Although we use the multiscale finite element framework, a finite volume element method (FVEM) is used for the global solution. This method is applied because, by construction, finite volume formulations satisfy local mass conservation, which is important for modeling transport in groundwater and reservoir flow simulations.

The underlying multiscale finite volume element method (MsFVEM) applied here is very similar to the multiscale finite volume technique of Jenny et al. $[15,16]$, though it differs in some details (e.g., location of pressure un- 
knowns), as indicated below. The key difference between this work and previous studies, however, is in the boundary conditions imposed in the determination of the MsFVEM basis functions, particularly the use of global coarse scale information for the specification of boundary conditions (in $[15,16]$ "reduced" boundary conditions, determined from the solution of lower-dimensional local problems, were applied). The local-global procedure applied here for this purpose does not appear to have been used previously within the context of multiscale finite element or finite volume models of subsurface flow.

\subsubsection{Basic multiscale finite volume element procedure}

The description and notation used here follow closely that of [9]. In [9], global information is also applied in the determination of the multiscale basis functions, but in that study fine scale data are used and no oversampling or iteration is required.

The basic problem is defined as follows (see Fig. 2). An underlying fine scale permeability description is specified. This description is considered to be too detailed for direct simulation of the pressure equation (3). We denote by $\mathcal{K}^{h}$ the set of coarse elements (rectangles in this case) $K$. The quantity $\boldsymbol{\xi}_{K}$ indicates the center of coarse element $K$. Element $K$ is divided into four rectangles of equal area by connecting $\boldsymbol{\xi}_{K}$ to the midpoints of the element edges. These quadrilaterals are denoted by $K_{\boldsymbol{\xi}}$, where $\boldsymbol{\xi} \in Z_{h}(K)$, are the vertices of $K$. We designate $Z_{h}=\bigcup_{K} Z_{h}(K)$ and $Z_{h}^{0} \subset Z_{h}$ the vertices which do not lie on the Dirichlet boundary of $\Omega$. The control volume $V_{\boldsymbol{\xi}}$ is defined as the union of the quadrilaterals $K_{\boldsymbol{\xi}}$ sharing the vertex $\boldsymbol{\xi}$. The grid comprised of elements $K$ (solid squares in Fig. 2) is sometimes referred to as the primal grid and the grid defined by $V_{\boldsymbol{\xi}}$ (dashed square in Fig. 2) as the dual grid. In our procedure we compute pressure at the vertices of the primal grid. This differs from the approach of $[15,16]$ in which pressure is computed at the centroids of the primal grid blocks. This also leads to a different treatment of global boundary conditions.

The goal of the MsFVEM is to determine coarse scale basis functions that incorporate the fine scale information in the underlying permeability description. The technique applied here follows the multiscale finite element method of [14], as the basis functions are determined from the solution of the leading order homogeneous elliptic equation on each coarse element. For a coarse rectangular element $K$, the basis functions $\phi_{i}, i=1,2,3,4$, are computed via solution of:

$$
\begin{aligned}
\nabla \cdot\left(\mathbf{k} \cdot \nabla \phi_{i}\right) & =0 \quad \text { in } K \\
\phi_{i} & =g_{i} \quad \text { on } \partial K
\end{aligned}
$$

for prescribed boundary function $g_{i}$. Equation (6) must be solved four times 


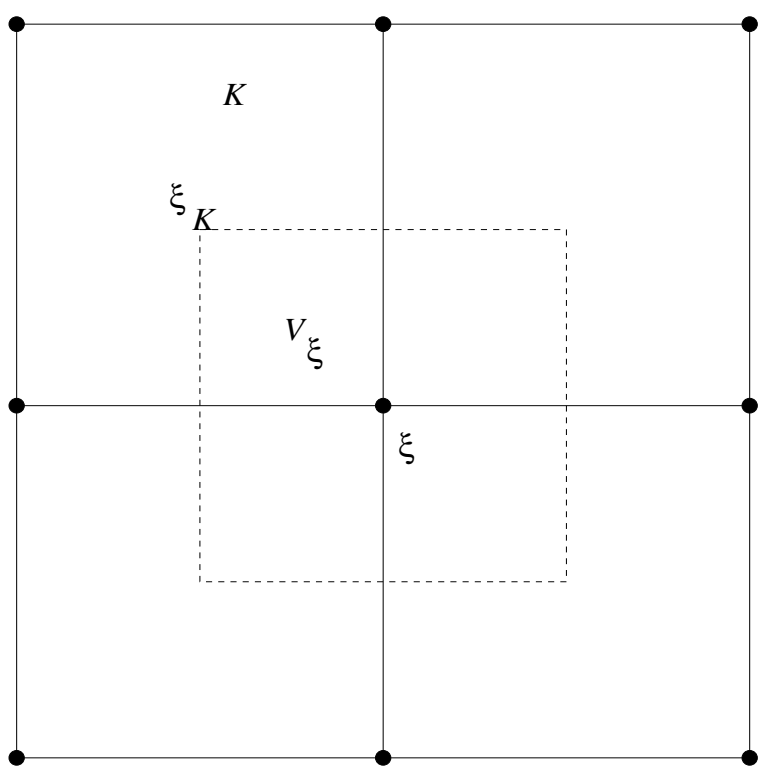

Fig. 2. Schematic of nodal points and grid.

for the determination of the four $\phi_{i}$. The basis function associated with the vertex $\mathbf{x}_{i}$ is constructed from the union of the basis functions that share this $\mathbf{x}_{i}$ and are zero elsewhere. Note that $\phi_{i}$ must satisfy $\phi_{i}\left(\mathbf{x}_{j}\right)=\delta_{i j}$.

Hou et al. [14] showed that the accuracy of the resulting coarse model is impacted by the treatment of boundary effects in (6). Enhanced accuracy can be achieved by solving local one-dimensional problems [15] for the determination of $g_{i}$ or, as is considered here, by solving (6) in a domain that includes more than just the fine scale cells corresponding to the coarse block $K$ (this approach is referred to as oversampling). The specific boundary conditions that are used in this paper for the determination of the basis functions will be discussed in detail below. A vertex-centered finite volume procedure is used to solve (6). The interface permeabilities, as required for this solution, are computed via a harmonic average of the appropriate component of permeability in the neighboring cells.

As discussed in [9], once the basis functions are constructed we determine $p^{h} \in V^{h}$, where $V^{h}$ is the space of the approximate pressure solution, with $p^{h}=\sum_{\mathbf{x}_{j} \in Z_{h}^{0}} p_{j} \phi_{j}$, by enforcing

$$
\int_{\partial V_{\boldsymbol{\xi}}}\left(\lambda(S) \mathbf{k} \cdot \nabla p^{h}\right) \cdot \mathbf{n} d l=\int_{V_{\boldsymbol{\xi}}} q d \mathbf{x}
$$

for every control volume $V_{\boldsymbol{\xi}} \subset \Omega$. Here $\mathbf{n}$ defines the normal vector on the boundary of the control volume $\partial V_{\boldsymbol{\xi}}$ and $S$ is the fine scale saturation. Note that the integral in $(7)$ is performed over a coarse cell in the dual grid $\left(V_{\boldsymbol{\xi}}\right)$ and the finite element test function is unity. For this reason, the technique is referred to as a finite volume element method. In this way the method differs 
from multiscale finite element procedures (e.g., [14]).

\subsubsection{Local-global iteration}

The procedure as defined up to this point (designated MsFVEM) is quite effective for many cases. However, in analogy to the upscaling results referred to above (and presented in $[6,5]$ ), some error is incurred due to the (localized) approximation of $g_{i}$ in (6). As demonstrated in [14-16], accurate results can be achieved through a judicious choice of $g_{i}$ and/or through use of oversampling. Each of these approaches, however, has some limitations. Oversampling leads to a nonconforming representation (as discussed below) and in addition does not account fully for global effects. The use of $g_{i}$ determined from the solution of a reduced (one-dimensional) problem has been shown to be effective $([14,15])$, though the ideal boundary specification is likely to be case specific. Again, in cases where global effects are important, it may be of use to incorporate this information directly into the basis functions.

We now describe an adaptive local-global procedure that introduces global information into the multiscale finite element basis functions. The resulting technique is our adaptive local-global multiscale finite volume element method, designated ALG-MsFVEM. The iteration procedures discussed here can be used within the context of either conforming or nonconforming multiscale finite volume element methods. A conforming procedure is one where the basis functions are continuous between the two elements that share $\partial K$. In a nonconforming representation, the basis functions are defined separately for each element and are in general discontinuous across $\partial K$. Conforming methods have some advantages over nonconforming techniques and may be preferable in some cases. For example, pressure is continuous at element interfaces with conforming methods, which is useful in compressible models where properties are pressure dependent. A correction must also be applied to obtain conservative velocities with the nonconforming method (as indicated below). In addition, analysis is more readily accomplished with conforming techniques.

More computation is required to construct the ALG-MsFVEM basis functions than is required for the MsFVEM approach described in Section 3.2.1. Depending on the size of the oversampling region, the construction of the ALG-MsFVEM basis functions could require several times more CPU time than that needed for the construction of MsFVEM basis functions. We expect that this extra computational demand could be reduced to some extent through use of thresholding (though this was not implemented here). For the coarse scale simulations, computational requirements are essentially the same for the two approaches. With either MsFVEM or ALG-MsFVEM, for typical problems the overhead associated with the construction of the basis functions is however small compared to the solution of the fine scale two-phase flow 
problem. This is because, due to mobility effects, the pressure equation (3) must be solved at each time step. It is therefore cost-effective to expend more computational effort (as required for ALG-MsFVEM) in forming the basis functions for the coarse model. It may not, however, be cost effective to perform multiple iterations of the ALG-MsFVEM, unless thresholding is applied. In addition, in either case computations for the construction of the basis functions parallelize naturally as they essentially involve independent solutions of (6) over small domains (i.e., domains corresponding to coarse elements or to coarse elements plus the oversampling region).

We first consider the development of a conforming ALG-MsFVEM procedure. In analogy to the ALG transmissibility upscaling described in Section 3.1, an estimate of the global solution is needed here for the determination of improved boundary conditions. This is accomplished by applying an oversampling procedure to determine the basis functions and then solving the global problem using these basis functions (note that the first step of the procedure generates nonconforming $\phi_{i}$, but the final representation will be conforming, as shown below). The procedure is as follows. Denote a target coarse block by $K$ and an extended coarse region by $K^{\prime}$ (see Fig. 3). For $K^{\prime}$ with vertices $\mathbf{y}_{i}$ $(i=1,2,3,4)$, we denote by $\psi_{i}(\mathbf{x})$ a nodal basis on $K^{\prime}$, such that $\psi_{i}\left(\mathbf{y}_{j}\right)=\delta_{i j}$. These nodal basis functions $\psi_{i}(i=1,2,3,4)$ are constructed by solving (6) in the region $K^{\prime}$ (see Fig. 3) with linear boundary conditions. Once the auxiliary functions $\psi_{i}$ are constructed, we compute the basis functions $\phi_{i}$ as a linear combination of $\psi_{i}$ (as is done in oversampling for MsFEM [14]) as follows:

$$
\phi_{i}(\mathbf{x})=\sum_{j=1}^{4} c_{i j} \psi_{j}(\mathbf{x})
$$

where $\mathbf{x}_{j}$ are the nodes of the target coarse block $K$ and $c_{i j}$ are coefficients determined by imposing $\phi_{i}\left(\mathbf{x}_{j}\right)=\delta_{i j}$. Using these nonconforming basis functions, the global problem is solved using (7).

We denote $p_{0}^{h}(\mathbf{x})$ as the solution of the pressure equation obtained as described above (using the MsFVEM with oversampling). In analogy to ALG upscaling, the adaptive local-global multiscale basis functions, $\Psi_{i}$, are constructed in target coarse regions using $p_{0}^{h}$ (note that these $\Psi_{i}$ are different from the oversampled $\psi_{i}$ defined above). Our approach (and description) again follow closely that of [9] except here we use the coarse scale solution $p_{0}^{h}(\mathbf{x})$ rather than the full fine scale solution as in [9]. At the edges where $\Psi_{i}(\mathbf{x})=0$ at both vertices, we take boundary conditions for $\Psi_{i}(\mathbf{x})$ to be zero. To compute $\Psi_{i}$ we now need to prescribe the boundary condition $g_{i}$ along the two edges that share the common vertex $\mathbf{x}_{i}$ (these edges are designated $\left[\mathbf{x}_{i-1}, \mathbf{x}_{i}\right]$ and $\left[\mathbf{x}_{i}, \mathbf{x}_{i+1}\right]$ ). Specifically, in analogy to [9] (and assuming that $p_{0}^{h}\left(\mathbf{x}_{i}\right) \neq p_{0}^{h}\left(\mathbf{x}_{i+1}\right)$ and $\left.p_{0}^{h}\left(\mathbf{x}_{i}\right) \neq p_{0}^{h}\left(\mathbf{x}_{i-1}\right)\right)$ we set: 


$$
\left.g_{i}(\mathbf{x})\right|_{\left[\mathbf{x}_{i}, \mathbf{x}_{i+1}\right]}=\frac{p_{0}^{h}(\mathbf{x})-p_{0}^{h}\left(\mathbf{x}_{i+1}\right)}{p_{0}^{h}\left(\mathbf{x}_{i}\right)-p_{0}^{h}\left(\mathbf{x}_{i+1}\right)},\left.\quad g_{i}(\mathbf{x})\right|_{\left[\mathbf{x}_{i}, \mathbf{x}_{i-1}\right]}=\frac{p_{0}^{h}(\mathbf{x})-p_{0}^{h}\left(\mathbf{x}_{i-1}\right)}{p_{0}^{h}\left(\mathbf{x}_{i}\right)-p_{0}^{h}\left(\mathbf{x}_{i-1}\right)}
$$

If $p_{0}^{h}\left(\mathbf{x}_{i}\right)=p_{0}^{h}\left(\mathbf{x}_{i+1}\right) \neq 0$ then

$$
\left.g_{i}\right|_{\left[\mathbf{x}_{i}, \mathbf{x}_{i+1}\right]}=\Psi_{i}^{0}(\mathbf{x})+\frac{1}{2 p_{0}^{h}\left(\mathbf{x}_{i}\right)}\left(p_{0}^{h}(\mathbf{x})-p_{0}^{h}\left(\mathbf{x}_{i+1}\right)\right)
$$

where $\Psi_{i}^{0}(\mathbf{x})$ is a linear function on $\left[\mathbf{x}_{i}, \mathbf{x}_{i+1}\right]$ such that $\Psi_{i}^{0}\left(\mathbf{x}_{i}\right)=1$ and $\Psi_{i}^{0}\left(\mathbf{x}_{i+1}\right)=0$. Similarly,

$$
\left.g_{i+1}\right|_{\left[\mathbf{x}_{i}, \mathbf{x}_{i+1}\right]}=\Psi_{i+1}^{0}(\mathbf{x})+\frac{1}{2 p_{0}^{h}\left(\mathbf{x}_{i+1}\right)}\left(p_{0}^{h}(\mathbf{x})-p_{0}^{h}\left(\mathbf{x}_{i+1}\right)\right),
$$

where $\Psi_{i+1}^{0}(\mathbf{x})$ is a linear function on $\left[\mathbf{x}_{i}, \mathbf{x}_{i+1}\right]$ such that $\Psi_{i+1}^{0}\left(\mathbf{x}_{i+1}\right)=1$ and $\Psi_{i+1}^{0}\left(\mathbf{x}_{i}\right)=0$. If $p_{0}^{h}\left(\mathbf{x}_{i}\right)=p_{0}^{h}\left(\mathbf{x}_{i+1}\right) \neq 0$, then linear boundary conditions can simply be applied. If $p_{0}^{h}\left(\mathbf{x}_{i}\right)=p_{0}^{h}\left(\mathbf{x}_{i+1}\right)=0$, linear boundary conditions can also be used (we note that this use of linear boundary conditions is somewhat akin to the treatment applied in ALG upscaling where precautions must be taken to avoid the calculation of anomalous $\left.T^{*}\right)$. Finally, the conforming ALGMsFVEM basis functions $\Psi_{i}$ are constructed by solving (6) in $K$ with $\Psi_{i}$ replacing $\phi_{i}$.

As in ALG transmissibility upscaling, we can perform additional iterations of this procedure to achieve self-consistency between the pressure field and $\Psi_{i}$. Generally the first iteration decreases the error most significantly, though further improvement can be obtained in some cases through another one or two iterations. In the results presented below, only one iteration is performed.

It is important to note that, as demonstrated below, the ALG basis functions $\Psi_{i}$ do not lie in the span of the first set of basis functions constructed using linear boundary conditions with oversampling. The $\Psi_{i}$ are linearly independent (and thus form a basis) and sum to 1 in each coarse element, except in the elements where $p_{0}^{h}\left(\mathbf{x}_{i}\right)=p_{0}^{h}\left(\mathbf{x}_{i+1}\right) \neq 0$ (in which case one basis function can be modified to ensure that the sum is equal to one; see [9]).

\subsubsection{Nonconforming ALG-MsFVEM}

It is also possible to apply the ALG procedure for the determination of nonconforming $\Psi_{i}$. The modifications required in this case are as follows. Following the solution of (6) over $K^{\prime}$ and the determination of $\phi_{i}$ from $\psi_{i}$ via (8), we solve the global coarse problem as above to give $p_{0}^{h}(\mathbf{x})$. Then, rather than using this $p_{0}^{h}(\mathbf{x})$ to determine boundary conditions along $\partial K$, we use $p_{0}^{h}(\mathbf{x})$ to set boundary conditions over a region larger than $K$, which we designate $\hat{K}$ 


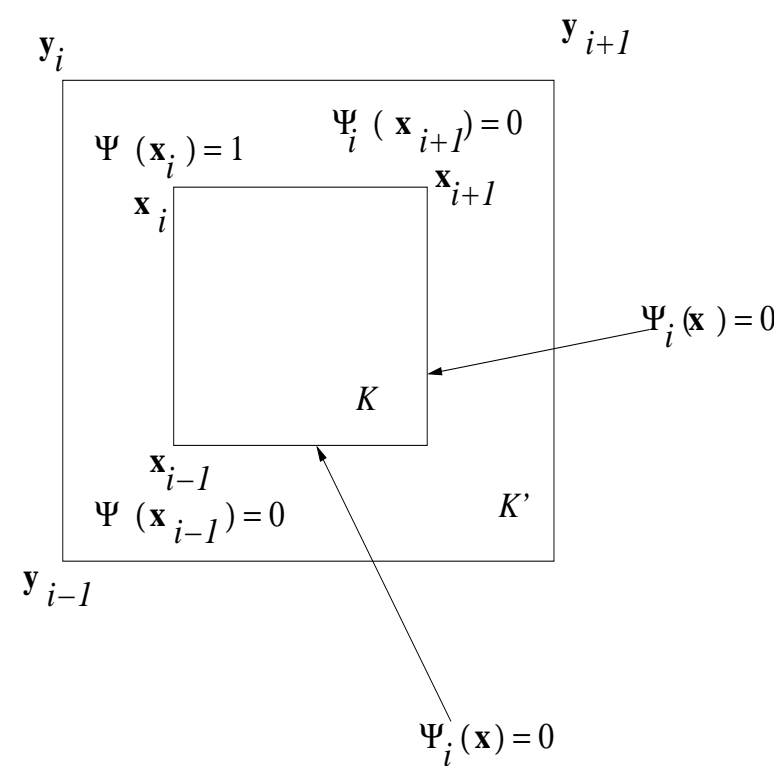

Fig. 3. Schematic description of coarse block and extended coarse block regions.

(this region may or may not coincide with $K^{\prime}$ ). More specifically, designating two edges of $\hat{K}$ as $\left[\mathbf{z}_{i-1}, \mathbf{z}_{i}\right]$ and $\left[\mathbf{z}_{i}, \mathbf{z}_{i+1}\right]$, the boundary conditions $g_{i}$ required for the determination of $\Psi_{i}$ are given through use of (9), (10) and (11) with $\mathbf{z}$ replacing $\mathbf{x}$. Solution of (6) subject to these $g_{i}$ and subsequent application of (8) (with $\Psi_{i}$ replacing $\phi_{i}$ ) gives the nonconforming ALG-MsFVEM basis functions. In the computations below, we take $K^{\prime}$ and $\hat{K}$ to coincide, though this is not required by the method.

A conservative fine scale velocity field is required for accurate transport calculations. If the $\Psi_{i}$ are nonconforming, their direct use for velocity reconstruction will lead to a nonconservative fine scale velocity field due to discontinuities in the basis functions along the boundaries of the coarse blocks. A conservative velocity can, however, be constructed by averaging the velocities from the two adjacent elements at the block boundaries.

\subsubsection{Two-phase flow computations}

For two-phase flow simulations, we first solve the coarse scale pressure equation (7) using the $\Psi_{i}$ (or $\phi_{i}$ in the case of the standard method). The fine scale velocity is then reconstructed by solving a local fine scale problem over each dual cell $V_{\boldsymbol{\xi}}$ with flux boundary conditions, as determined from the pressure solution and the $\Psi_{i}$, prescribed. This velocity is then used in the explicit solution of the saturation equation (4). The overall procedure is thus an IMPES (implicit pressure, explicit saturation) approach.

With ALG-MsFVEM, the pressure equation is solved using the pre-computed multiscale basis functions $\Psi_{i}$. In some multiscale simulation procedures (e.g., 
[16]), the basis functions are updated in time in the vicinity of sharp fronts. In our calculations, we observed only a slight improvement from this updating. Therefore, in the results below, the basis functions are not updated. The update of the basis functions is, however, performed if the global boundary conditions are changed. In this way the ALG-MsFVEM "adapts" to the particular global boundary specification. As indicated earlier, the ALG framework allows us to perform updates in an efficient manner; i.e., we only recompute the basis functions in selected regions (see [5] for details). This thresholding is compatible with the ALG-MsFVEM formulation but was not used in the results presented here.

\subsubsection{Explanation of ALG-MsFVEM accuracy}

We now offer a brief explanation of why the proposed method generally performs better than the standard MsFVEM with localized basis functions for the case of two-phase flow in a strongly channelized formation. As shown in [9], the pressure equation (3) can be written in a coordinate system defined by the streamfunction $\psi$ and the pressure $p$ at initial time; i.e., $\eta=\psi(\mathbf{x}, t=0)$ and $\zeta=p(\mathbf{x}, t=0)$. Assuming that the the permeability variation within the channel (in the $\eta$ direction) is weak, that $S=0$ at initial time, and that the imposed global boundary conditions result in high flow within the channel (this is fairly typical), it was shown in [9] that

$$
p(\eta, \zeta, t)=\hat{p}(\zeta, t)+\text { high order terms, }
$$

where $\hat{p}(\zeta, t)$ is the dominant pressure.

This result indicates that the pressure (which varies in time due to saturation effects) depends strongly on the initial pressure $\zeta$; i.e., the leading order term in the asymptotic expansion is a function of initial pressure and time only. Thus, if the multiscale basis functions are able to recover the initial pressure field with reasonable accuracy, they can then be expected to provide an accurate estimate of pressure throughout the simulation (at least for the idealized situation considered here). The ALG-MsFVEM basis functions carry global information as they are constructed from the global solution, so we can expect reasonable accuracy for pressure at initial time and thus throughout the simulation. We will see this to be the case in the results presented in the next section.

As indicated above, the ALG basis functions $\Psi_{i}$ contain global information and, as such, do not lie in the span of $\phi_{i}$. Indeed, assuming $\Psi_{i}$ lie in span of $\phi_{i}$, we would conclude that $\Psi_{i}=\phi_{i}$. To show that $\Psi_{i} \neq \phi_{i}$, we consider, for example, the edge $\left[\mathbf{x}_{i}, \mathbf{x}_{i+1}\right]$ (see Fig. 3), where both $\phi_{i}$ and $\Psi_{i}$ are 1 and 0 at $\mathbf{x}_{i}$ and $\mathbf{x}_{i+1}$, respectively. The function $\phi_{i}$ on $\left[\mathbf{x}_{i}, \mathbf{x}_{i+1}\right]$ is computed using $\psi_{i}$, while $\Psi_{i}$ on $\left[\mathbf{x}_{i}, \mathbf{x}_{i+1}\right]$ is computed from (9) using $p_{0}^{h}=\sum_{i} p_{0}^{h}\left(\mathbf{x}_{i}\right) \phi_{i}$. More 
precisely, if $p_{0}^{h}\left(\mathbf{x}_{i+1}\right) \neq p_{0}^{h}\left(\mathbf{x}_{i}\right)$,

$$
g_{i}=\frac{p_{0}^{h}\left(\mathbf{x}_{i}\right) \phi_{i}(\mathbf{x})+p_{0}^{h}\left(\mathbf{x}_{i+1}\right) \phi_{i+1}(\mathbf{x})-p_{0}^{h}\left(\mathbf{x}_{i+1}\right)}{p_{0}^{h}\left(\mathbf{x}_{i}\right)-p_{0}^{h}\left(\mathbf{x}_{i+1}\right)} .
$$

It is clear that $p_{0}^{h}\left(\mathbf{x}_{i}\right)$ enters into $\Psi_{i}$ in a nonlinear fashion, $\Psi_{i} \neq \phi_{i}$, and that the $\Psi_{i}$ contain global information from $p_{0}^{h}$. The global information contained in $p_{0}^{h}$ is however not as accurate as the information contained in the exact initial fine scale pressure field. If the basis functions can recover the exact initial pressure field, more accurate multiscale solutions can be expected.

\section{Numerical results}

We now present results for several heterogeneous systems. In these simulations, dimensionless pressures are fixed along portions of the boundary ( $p=1$ at inflow boundaries and $p=0$ at production boundaries) and no-flow conditions are imposed over the remainder. We consider both single-phase and two-phase flow simulations. For single-phase simulations, results are presented only for total flow rate $Q$.

For the two-phase flow simulations, the system is considered to initially contain only oil $(S=0)$ and water is injected at inflow boundaries $(S=1$ is prescribed). Relative permeability functions are specified as $k_{r w}=S^{2}$, $k_{r o}=(1-S)^{2}$; water and oil viscosities are set to $\mu_{w}=1$ and $\mu_{o}=5$. Porosity is constant and serves only to nondimensionalize time. Results are presented in terms of total flow rate $Q$ and the fraction of oil in the produced fluid (i.e., oil cut, designated $F$ ) against pore volume injected (PVI). PVI represents dimensionless time and is computed via $\int Q d t / V_{p}$ where $V_{p}$ is the total pore volume of the system. In all cases we compare solutions using the standard multiscale procedure (MsFVEM) and the adaptive local-global multiscale technique (ALG-MsFVEM) to the reference fine scale solution. By standard MsFVEM calculations, we mean that no oversampling is used and linear boundary conditions are applied. The use of nonconforming (oversampled) representations is considered in Section 4.2. We note that other local boundary conditions could be applied $([14,15])$ and may provide better accuracy in some cases, though the ideal boundary specification is likely to be case specific. The development and use of specialized local boundary conditions is, however, not investigated here.

We consider channelized permeability fields extracted from the three-dimensional system introduced in [8]. This highly heterogeneous system has been considered in a number of previous studies (e.g., [5]) and poses a challenge for upscaling and multiscale procedures. Channelized systems of this type are con- 
siderably more difficult to model accurately than are systems generated using two-point (variogram-based) geostatistics. Thus, methods that perform accurately for the channelized systems considered here can be expected to perform as well or better on simpler variogram-based models.

The fine grid models are of dimension $220 \times 60$. Two typical layers are shown in Fig. 4. In all simulations, we take the global dimension $(L)$ in $x$ and $y$ to be $L_{x}=5$ and $L_{y}=1$, with cell sizes $\Delta x=5 / 220$ and $\Delta y=1 / 60$, unless otherwise indicated. The coarse grid in all cases is of dimension $22 \times 6$ (a coarsening factor of 10 in each direction).

\subsection{Results for conforming MsFVEM and ALG-MsFVEM}

The results presented in this section compare standard MsFVEM and conforming ALG-MsFVEM solutions. In the ALG-MsFVEM simulations, an oversampling of five fine scale cells is applied in the first step. The conforming representation is achieved in the iteration step as described in Section 3.2.2.

In the first set of simulations, we treat each of the channelized layers in the three-dimensional model (these are the lower 50 layers) as a two-dimensional system and simulate flow across the layer by imposing $p=1$ along $x=0$ and $p=0$ along $x=5$. For these simulations, the system is single-phase and the flow rates do not change in time. Presented in Fig. 5 (left) is a cross plot of the coarse scale $Q$, computed using the standard MsFVEM, against the fine scale result. The average relative error (defined as the average of the percentage errors of all 50 points) in these results is $29.3 \%$ and there is a clear trend toward overprediction by the standard MsFVEM (it should be noted that, if we remove the point with the maximum error, designated by the $\times$ in Fig. 5left, the average relative error is reduced to 17.8\%). Analogous results using the ALG-MsFVEM are shown in Fig. 5 (right). These results are noticeably improved relative to those of the standard technique; the average relative error here is $1.8 \%$. This clearly demonstrates the efficacy of the ALG-MsFVEM and that the use of even approximate global information can act to enhance the accuracy of the multiscale solution.

We next consider two-phase flow for layer 43 (with relative permeabilities and viscosities as specified above). Flow is from side to side and boundary conditions are as prescribed in the previous case. The total flow rate $Q$ now varies in time due to mobility effects. Results for $F$ and $Q$ are shown in Fig. 6 . The solid curves represent the fine scale solution and the dashed and dotted curves the standard MsFVEM and ALG-MsFVEM solutions respectively. The ALG procedure is seen to provide improved accuracy, though there is still some error relative to the fine scale solution. At the end of the simulation $(\mathrm{PVI}=2)$, 

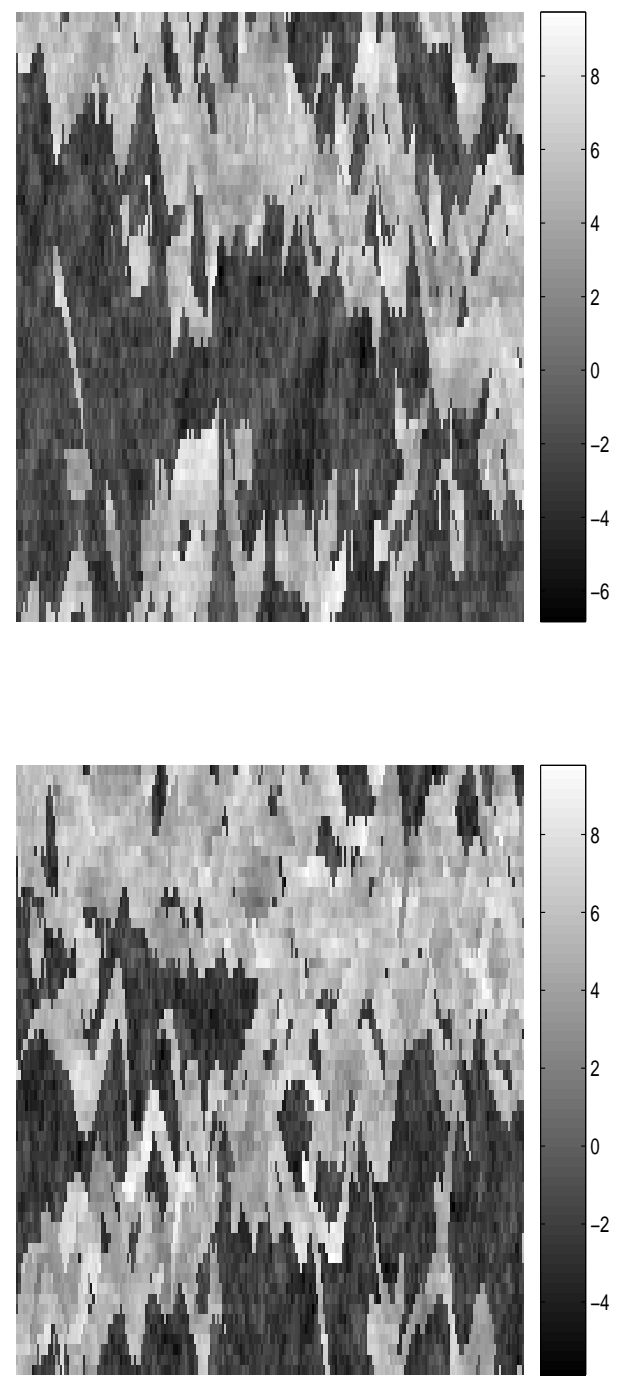

Fig. 4. Permeability fields (from [8]) for layers 43 (left) and 59 (right). Vertical direction exaggerated.

the standard MsFVEM shows an $11.5 \%$ error in flow rate; this error is reduced to $3.5 \%$ through use of ALG-MsFVEM.

It is also instructive to consider the saturation maps provided by the various procedures. The fine scale saturation field at PVI $=0.4$ is shown in Fig. 7 (left). It is evident that the flow is dominated by the high permeability channel extending from the upper left of the model (see Fig. 4). The saturation map for the standard MsFVEM is shown in Fig. 7 (center). The general appearance of this result is quite similar to the fine scale result, though there are differences evident in the lower left corner of the model, where there is a disconnected high permeability feature (evident in Fig. 4). The ALG-MsFVEM result is shown in Fig. 7 (right). The general correspondence between this map and 

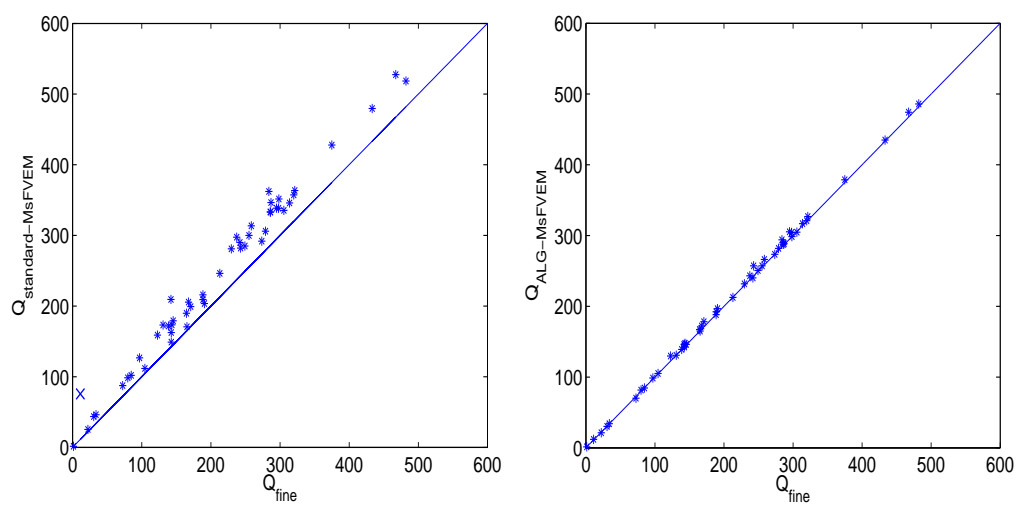

Fig. 5. Comparison of global flow rates for side to side flow for standard MsFVEM (left) and ALG-MsFVEM (right).
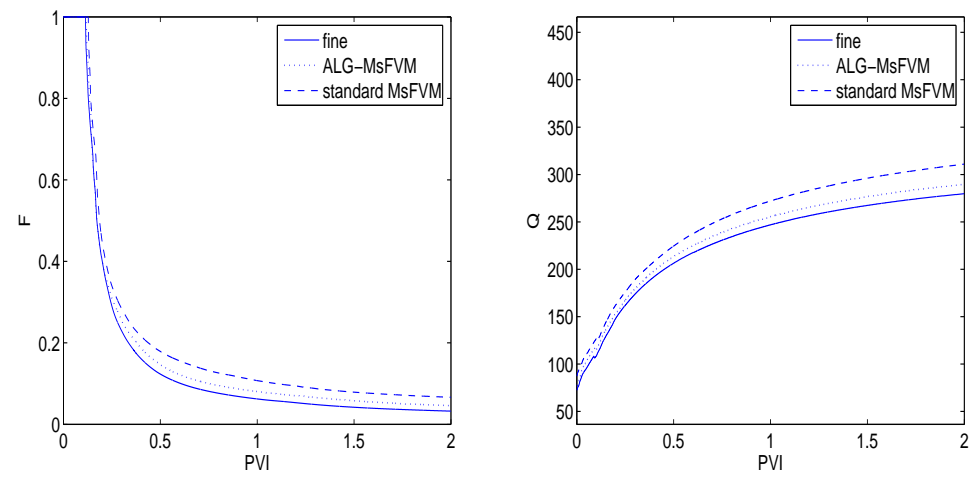

Fig. 6. Oil cut (left) and total flow rate (right) for layer 43 for side to side flow.

the fine scale result is again quite close, though in this case there is less error in the lower left corner of the model. This is because the "disconnectedness" of the permeability feature is captured more accurately by the ALG-MsFVEM model than it is by the standard procedure, for which the basis functions are computed using only local information.

In the next set of results, we consider different boundary conditions (corner to corner rather than side to side flow) and perform single-phase flow calculations for all 50 channelized layers. For this case, we set $p=1$ along $x=0,0 \leq y \leq$ 0.4 and $p=0$ along $x=5,0.6 \leq y \leq 1$. The ALG-MsFVEM model will be different in this case than in the side to side flow case because the basis functions depend on the global flow field. Results for the standard MsFVEM are shown in Fig. 8 (left) and those for ALG-MsFVEM are shown in Fig. 8 (right). The average relative errors for the standard and ALG procedures are $25.7 \%$ and $7.5 \%$ respectively. The error using ALG-MsFVEM is larger in this case than it is for side to side flow (compare to Fig. 5). This may be due to the fact that the flow is confined to smaller portions of the domain in corner to corner flow and the results are sensitively dependent on the accurate resolution of the solution in these regions. 

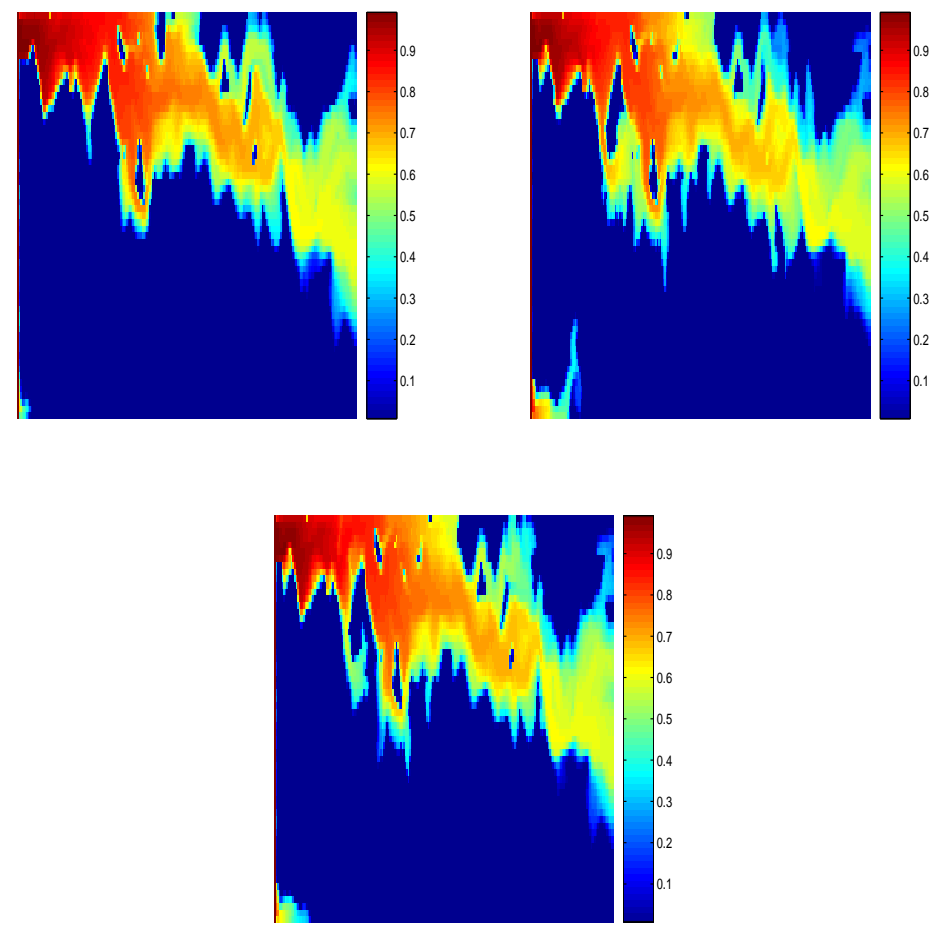

Fig. 7. Saturation maps for layer 43 for side to side flow for fine model (left), standard MsFVEM (center) and ALG-MsFVEM (right). Vertical direction exaggerated.
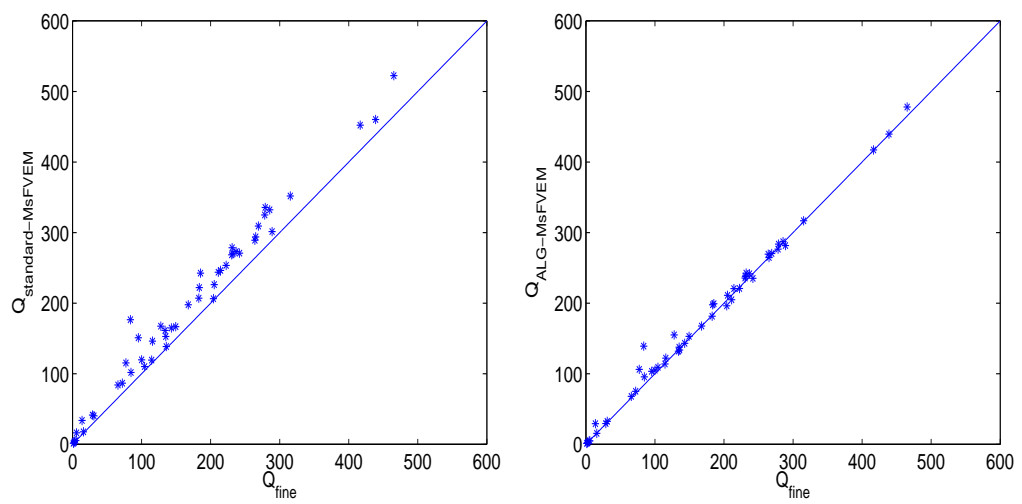

Fig. 8. Comparison of global flow rates for corner to corner flow for standard MsFVEM (left) and ALG-MsFVEM (right).

We now display results for two-phase corner to corner flow for layer 43. Results for oil cut and total flow rate are shown in Fig. 9. The use of ALG-MsFVEM offers some improvement in this case but the level of agreement with the fine scale solution is not as high as for side to side flow. This observation is consistent with the single-phase flow results presented above.

We next consider both types of flow (side to side and corner to corner) for layer 59. Results are displayed in Figs. 10 and 11. For this layer, the use of 

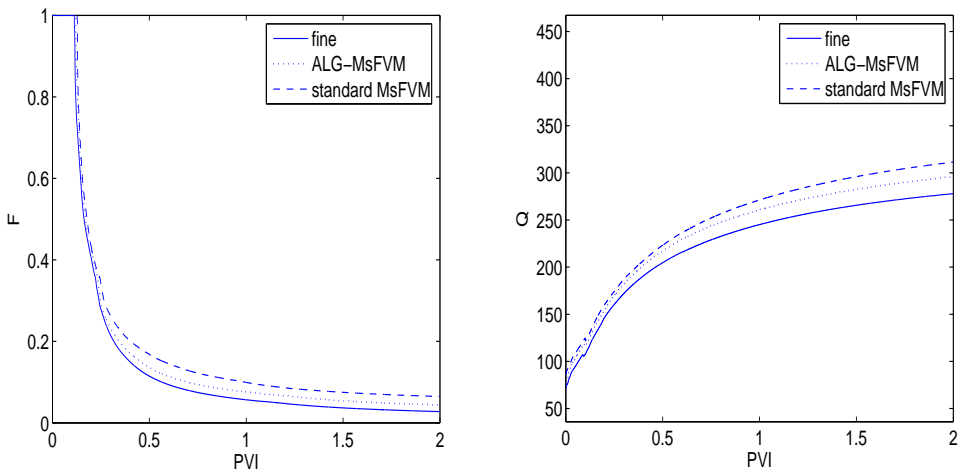

Fig. 9. Oil cut (left) and total flow rate (right) for layer 43 for corner to corner flow.
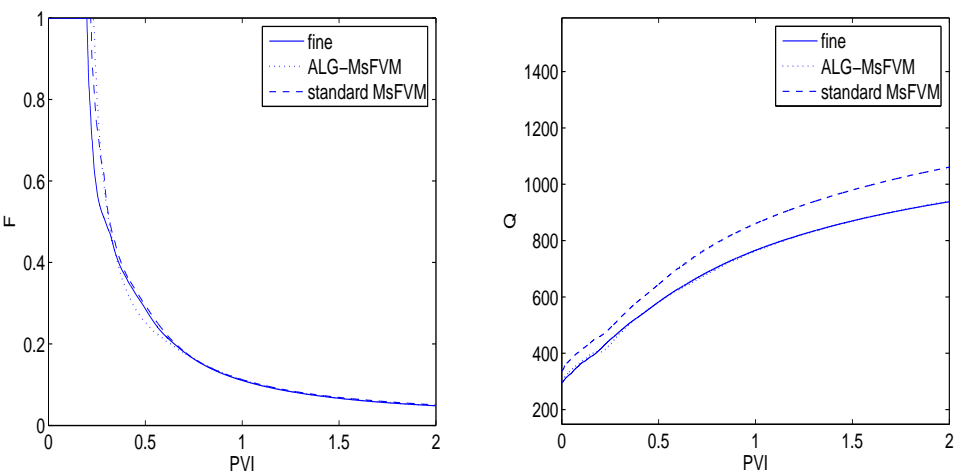

Fig. 10. Oil cut (left) and total flow rate (right) for layer 59 for side to side flow.
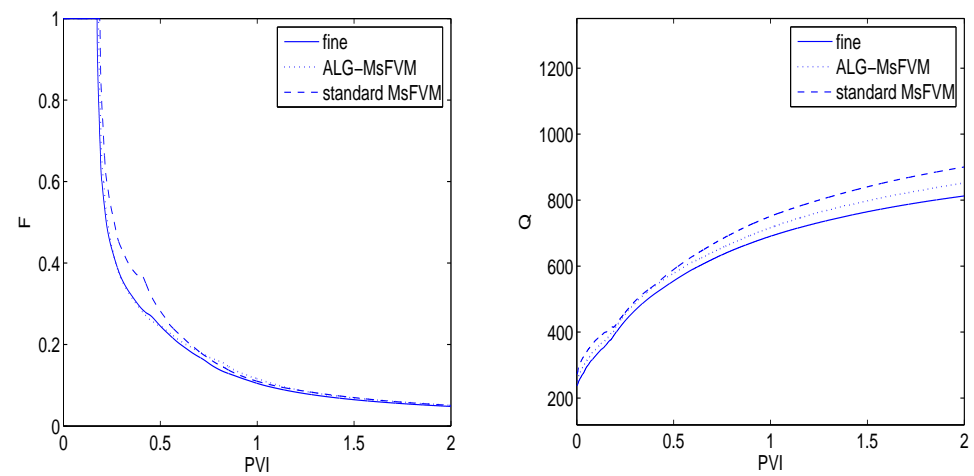

Fig. 11. Oil cut (left) and total flow rate (right) for layer 59 for corner to corner flow.

ALG-MsFVEM provides an excellent match with the fine scale solution for side to side flow (Fig. 10), in contrast to the standard MsFVEM which shows some error (about a $12 \%$ error in $Q$ at $\mathrm{PVI}=2$ ). For corner to corner flow, the level of agreement with ALG-MsFVEM is not as close, though better accuracy is again attained with this method than with the standard procedure.

Our next example involves a change in flow conditions during the course of 

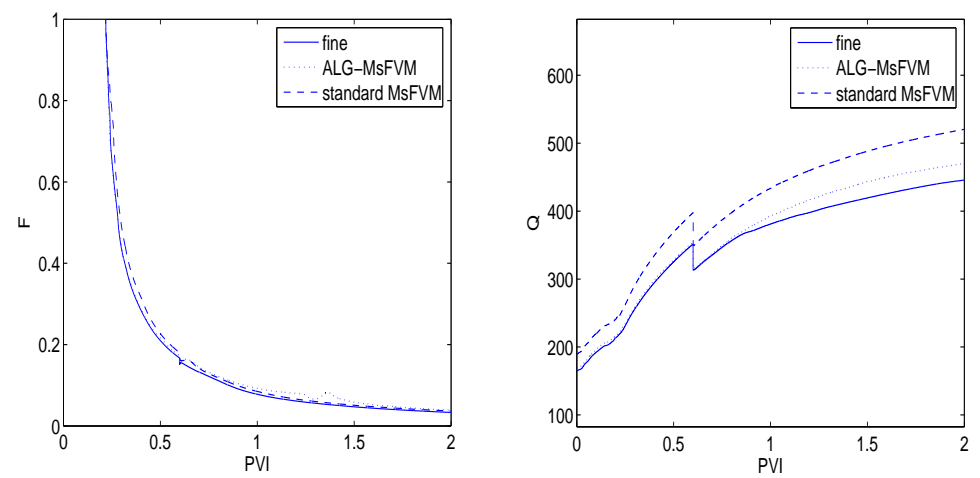

Fig. 12. Oil cut (left) and total flow rate (right) for layer 63. Side to side flow for $\mathrm{PVI} \leq 0.6$ followed by corner to corner flow for PVI $>0.6$.

the simulation. Specifically, the flow is from side to side for PVI $\leq 0.6$ and from corner to corner for PVI $>$ 0.6. Results for layer 63 are shown in Fig. 12. The ALG-MsFVEM solution shows excellent accuracy for PVI $\leq 0.6$, though there is some degradation in accuracy after the change in boundary conditions. The ALG-MsFVEM solution is, however, clearly of greater accuracy than the standard MsFVEM solution. It is important to note that the ALG-MsFVEM basis functions are recomputed after the change in boundary conditions, which enables the method to adapt to the change in the global flow field.

We also explored the use of additional iterations in the ALG-MsFVEM. A limited study indicated that solution accuracy can generally be improved by performing more iterations. However, to be cost effective and robust, this procedure should ideally be applied in conjunction with thresholding. There are a variety of strategies that could be used, and a detailed evaluation will require further numerical experimentation.

\subsection{Results for nonconforming MsFVEM and ALG-MsFVEM}

In the results presented above, the MsFVEM was in all cases conforming, meaning that the basis functions $\phi_{i}$ or $\Psi_{i}$ are continuous between adjacent elements. We now consider the use of nonconforming representations. Recall that there are some potential disadvantages associated with nonconforming representations, as discussed above, though such models are nonetheless useful in many settings. The nonconforming representations considered here are generated in two ways, corresponding to the standard and ALG models considered earlier. Specifically, here we assess "standard" oversampling (without iteration, as applied in [14]) and oversampling used in conjunction with one local-global iteration. In the latter case, we use the same degree of oversampling in both the initial estimate of the basis functions and in the iteration using the global solution (i.e., $K^{\prime}$ and $\hat{K}$ are identical). This is not a require- 
ment of the method, and it is possible that other strategies will result in improved accuracy, as would additional local-global iteration.

In Table 1 we present results for average relative error in flow rate for side to side flow for the 50 channelized layers using various conforming and nonconforming procedures. Results are shown for both the model considered above (with global dimensions $L_{x} / L_{y}=5$ ) and for a stretched model with $L_{x} / L_{y}=20$, as indicated in the first column. The second column corresponds to the standard localized conforming method with linear pressure boundary conditions and no oversampling (these are the results presented in Fig. 5-left). The third column presents results using oversampling (ovs) of five fine-grid cells (with no local-global iteration), the fourth column corresponds to ALGMsFVEM results with oversampling of five fine-grid cells in both the initial estimate and in the ALG iteration, and the fifth column presents results using oversampling in the initial estimate but a conforming representation after iteration (these are the results presented in Fig. 5-right).

Table 1

MsFVEM and ALG-MsFVEM percentage errors in flow rate, with five fine grid cells used in oversampling

\begin{tabular}{|c|c|c|c|c|}
\hline$L_{x} / L_{y}$ & conform & ovs & ovs-ovs & ovs-conform \\
\hline $5 \times 1$ & $29.3 \%$ & $4.7 \%$ & $3.4 \%$ & $1.8 \%$ \\
\hline $20 \times 1$ & $28.7 \%$ & $10.1 \%$ & $6.3 \%$ & $6.7 \%$ \\
\hline
\end{tabular}

It is evident from the results presented in the table that oversampling without iteration provides improved accuracy (compare columns two and three), consistent with previous findings [14]. Application of an ALG iteration reduces the error, as is evident from the values in the fourth column, though improvement is modest in this case. For these results, local-global iteration is more beneficial when the goal is to provide a conforming set of basis functions (compare columns two and five). It is also apparent that accuracy degrades somewhat (except for the localized conforming method) for the case of $L_{x} / L_{y}=20$ compared to $L_{x} / L_{y}=5$.

In the next set of results, presented in Table 2, the same systems are considered but here we use oversampling of two fine grid cells rather than five, as applied in all of the previous results. Here we see that standard oversampling with two fine cells leads to less error reduction than in the case of five-cell oversampling, though an ALG iteration (fourth column) reduces this error by about a factor of two. The conforming ALG model (fifth column) is also not as accurate as in the five-cell oversampling (compare with Table 1) though it is more accurate than the standard conforming model. This suggests that the benefit of using ALG-MsFVEM is more fully realized when the global estimate is of better accuracy, as would be expected. In any event, the results in Tables 1 and 2 demonstrate that local-global iteration consistently acts to improve accuracy, 
for either conforming or nonconforming representations, though the degree of improvement varies depending on the size of the oversampling domain.

Table 2

MsFVEM and ALG-MsFVEM percentage errors in flow rate, with two fine grid cells used in oversampling

\begin{tabular}{|c|c|c|c|c|}
\hline$L_{x} / L_{y}$ & conform & ovs & ovs-ovs & ovs-conform \\
\hline $5 \times 1$ & $29.3 \%$ & $12.6 \%$ & $6.0 \%$ & $16.0 \%$ \\
\hline $20 \times 1$ & $28.7 \%$ & $18.8 \%$ & $9.3 \%$ & $21.0 \%$ \\
\hline
\end{tabular}

\section{Concluding remarks}

The purpose of this paper was to develop and apply an adaptive local-global multiscale finite volume element procedure for the modeling of two-phase flow in heterogeneous subsurface formations. The method effectively combines multiscale finite volume element and upscaling techniques to provide an efficient means for incorporating global information into the multiscale basis functions. This efficiency results from the use of coarse scale simulations of the global pressure equation for the determination of approximate global information.

Both the new method and a standard multiscale finite volume element method (which uses localized linear pressure boundary conditions for determination of the basis functions) were applied to a series of challenging example problems involving highly heterogeneous channelized permeability fields. Results for both single-phase and two-phase systems, involving different global boundary conditions, were presented. In all cases the ALG-MsFVEM provided results of improved accuracy, in terms of reproduction of the reference fine scale solution, compared to the standard technique.

As indicated earlier, improved accuracy can also be obtained through a judicious choice of local boundary conditions or through oversampling. In this paper we investigated oversampling and, consistent with previous findings (e.g., [14]), found it to reduce error relative to the standard procedure. When oversampling is used, however, the resulting MsFVEM is nonconforming, which results in discontinuities in the reconstructed pressure at coarse block boundaries. We did not investigate the use of other local boundary conditions (such as the "reduced" boundary conditions applied in [14,15]). The use of these or other specialized boundary conditions may well provide improved performance for the problems considered, though it is to be expected that the ideal boundary specification will depend on various factors such as the type of geological model, global boundary conditions and degree of coarsening. Our approach is in some sense more systematic, as we determine these boundary conditions 
from a global pressure solution. The development and assessment of appropriate local boundary conditions is, however, a useful endeavor, as this could provide a more efficient overall procedure. In addition, it would be straightforward to use specialized boundary conditions in the first iteration of the local-global procedure described here.

Although the results presented in this paper are encouraging, there is scope for further exploration of some of the underlying approaches. As our intent here was to demonstrate that global coarse scale information could be effectively used to improve multiscale representations, we did not incorporate time-saving procedures such as the thresholding technique incorporated in ALG upscaling. Introduction of this or a similar treatment into the ALG-MsFVEM will lead to improved efficiency and possibly enhanced robustness. It may also be worth exploring the impact of different oversampling strategies, additional ALG iterations, and the use of different boundary conditions in the initial estimate for the basis functions.

\section{References}

[1] J. AARNES, On the use of a mixed multiscale finite element method for greater flexibility and increased speed or improved accuracy in reservoir simulation, SIAM Multiscale Modeling and Simulation, 2 (2004), pp. 421-439.

[2] J. Aarnes, V. Kippe, And K.-A. Lie, Mixed multiscale finite elements and streamline methods for reservoir simulation of large geomodels, Advances in Water Resources, 28 (2005), pp. 257-271.

[3] T. Arbogast, Implementation of a locally conservative numerical subgrid upscaling scheme for two-phase Darcy flow, Computational Geosciences, 6 (2002), pp. 453-481.

[4] T. Arbogast and S. L. Bryant, A two-scale numerical subgrid technique for waterflood simulations, SPE Journal, 7 (2002), pp. 446-457.

[5] Y. Chen And L. J. Durlofsky, Adaptive local-global upscaling for general flow scenarios in heterogeneous formations, Transport in Porous Media, 62 (2006), pp. 157-185.

[6] Y. Chen, L. J. Durlofsky, M. Gerritsen, and X. H. Wen, A coupled local-global upscaling approach for simulating flow in highly heterogeneous formations, Advances in Water Resources, 26 (2003), pp. 1041-1060.

[7] Z. Chen And T. Y. Hou, A mixed multiscale finite element method for elliptic problems with oscillating coefficients, Mathematics of Computation, 72 (2003), pp. $541-576$. 
[8] M. Christie And M. Blunt, Tenth SPE comparative solution project: A comparison of upscaling techniques, SPE Reser. Eval. Eng., 4 (2001), pp. 308317.

[9] Y. Efendiev, V. Ginting, T. Hou, and R. Ewing, Accurate multiscale finite element methods for two-phase flow simulations. Submitted.

[10] Y. Efendiev, T. Hou, And V. Ginting, Multiscale finite element methods for nonlinear problems and their applications, Comm. Math. Sci., 2 (2004), pp. $553-589$.

[11] C. L. FARmer, Upscaling: a review, International Journal for Numerical Methods in Fluids, 40 (2002), pp. 63-78.

[12] M. Gerritsen and L. J. Durlofsky, Modeling of fluid flow in oil reservoirs, Annual Reviews in Fluid Mechanics, 37 (2005), pp. 211-238.

[13] L. Holden And B. F. Nielsen, Global Upscaling of Permeability in Heterogeneous Reservoirs: the Output Least Squares (OLS) Method, Transport in Porous Media, 40 (2000), pp. 115-143.

[14] T. Y. Hou AND X. H. Wu, A multiscale finite element method for elliptic problems in composite materials and porous media, Journal of Computational Physics, 134 (1997), pp. 169-189.

[15] P. Jenny, S. H. Lee, And H. TChelePi, Multi-scale finite volume method for elliptic problems in subsurface flow simulation, J. Comput. Phys., 187 (2003), pp. 47-67.

[16] P. Jenny, S. H. Lee, And H. TChelepi, Adaptive multi-scale finite volume method for multi-phase flow and transport in porous media, SIAM Multiscale Modeling and Simulation, 3 (2005), pp. 30-64.

[17] Ph. Renard and G. De Marsily, Calculating effective permeability: a review, Advances in Water Resources, 20 (1997), pp. 253-278.

[18] R. K. Romeu And B. Noetinger, Calculation of internodal transmissivities in finite difference models of flow in heterogeneous porous media, Water Resources Research, 31 (1995), pp. 943-959.

[19] X. H. Wen and J. J. Gómez-Hernández, Upscaling hydraulic conductivities in heterogeneous media: an overview, Journal of Hydrology, 183 (1996), pp. ixxxxii. 